\title{
Calculations Using Quantum Chemistry for Inorganic Molecule Simulation $\mathrm{BeLi}_{2} \mathrm{SeSi}$
}

\author{
Ricardo Gobato ${ }^{1, *}$, Alireza Heidari ${ }^{2}$ \\ ${ }^{1}$ State Secretariat for Education of Paraná, Laboratory of Biophysics and Molecular Modeling, Bela Vista do Paraíso, Paraná, Brazil \\ ${ }^{2}$ Faculty of Chemistry, California South University, Irvine, California, USA
}

\section{Email address:}

ricardogobato@seed.pr.gov.br (R. Gobato), Scholar.Researcher.Scientist@gmail.com (A. Heidari), Alireza.Heidari@calsu.us (A. Heidari) ${ }^{*}$ Corresponding author

\section{To cite this article:}

Ricardo Gobato, Alireza Heidari. Calculations Using Quantum Chemistry for Inorganic Molecule Simulation BeLi ${ }_{2} \mathrm{SeSi}_{\text {. Science Journal of }}$ Analytical Chemistry. Vol. 5, No. 5, 2017, pp. 76-85. doi: 10.11648/j.sjac.20170505.13

Received: June 20, 2017; Accepted: July 17, 2017; Published: October 18, 2017

\begin{abstract}
Inorganic crystals have been used in the most diverse electronic systems since the nineteenth century, which apply to the wide variety of technological applications, which are the quartz crystals are the most used. Elements such as beryllium, lithium, silicon and selenium are widely used. The difficulty of finding such crystals from the combination of elements in nature or synthesized, suggest an advanced study of the same. In this sense, these elements were chosen because of the physical-chemical properties of each one, to simulate a seed molecule whose arrangement would be formed by the combination of these, aiming at the future development of a crystal to be used technologically. A study using computer programs with $a b$ initio method was applied and the quantum chemistry was utilized through Molecular Mechanics, HartreeFock, Møller-Plesset and Density Functional Theory, on several bases. The main focus was to obtain a stable molecular structure acceptable to quantum chemistry. As a result of the likely molecular structure of the arrangement of a crystal was obtained, beyond the dipole moment, thermal energy, heat of vaporization and entropy of the molecule. The simulated molecule has a cationic molecular structure, in the atoms Selenium and Silicon. As a consequence, it has a strong electric dipole moment. Due to its geometry, it presents a probable formation structure of a crystal with a tetrahedral and hexahedral crystal structure.
\end{abstract}

Keywords: Beryllium, DFT, Lithium, Molecular Geometry, Selenium, Silicon

\section{Introduction}

A crystal [1] oscillator is an electronic oscillator circuit that uses the mechanical resonance of a vibrating crystal of piezoelectric material to create an electrical signal with a very precise frequency. This frequency is commonly used to keep track of time (as in quartz wristwatches), to provide a stable clock signal for digital integrated circuits, and to stabilize frequencies for radio transmitters and receivers. The most common type of piezoelectric resonator used is the quartz crystal, so oscillator circuits incorporating them became known as crystal oscillators, but other piezoelectric materials including polycrystalline ceramics are used in similar circuits. [2, 3]

The use of inorganic crystals technology has been widely date. Since quartz crystals [1] for watches in the nineteenth century, and common way radio in the early twentieth century, to computer chips with new semiconductor materials. [2] Chemical elements such as beryllium, lithium, selenium and silicon, [1, 4] are widely used in technology. The development of new crystals arising from that arrangement can bring technological advances in several areas of knowledge.

Within many electronics resonates a crystal that determines a precise rhythm functioning. The clocks, timers, computers, communications equipment and many other tiny devices quartz crystals [1] vibrate accurately ensuring that your circuits work completely orderly and synchronized way. It is difficult to predict what would be electronics today without the presence of these elements. [5]

A study using computer programs with ab initio method was applied and the quantum chemistry was utilized through Molecular Mechanics, Hartree-Fock, Møller-Plesset and 
Density Functional Theory, on several bases. [6, 7]

A preliminary literature search did not indicate any compounds of said arrangement of these chemical elements. This fact the study can lead to getting new crystals to be used in the materials industry. A study using computer programs with ab initio have been applied. The calculations indicate by the $a b$ initio methods used that the molecular structure of the simulated molecule can in more detailed calculations generate a crystalline structure. $[8,9]$

\section{Chemical Properties of the Compounds of Beryllium, Lithium, Selenium and Silicon}

The Beryllium, Lithium, Selenium and Silicon elements were chosen due to their peculiar physicochemical properties and their wide use in industry and technology.

\subsection{Beryllium}

Beryllium is a chemical element with symbol $\mathrm{Be}$ and atomic number 4 . It is created through stellar nucleosynthesis and is a relatively rare element in the universe. It is a divalent element which occurs naturally only in combination with other elements in minerals. Notable gemstones which contain beryllium include beryl (aquamarine, emerald) and chrysoberyl. As a free element it is a steel-gray, strong, lightweight and brittle alkaline earth metal. [2]

Beryllium improves many physical properties when added as an alloying element to aluminium, copper (notably the alloy beryllium copper), iron and nickel. [10] Tools made of beryllium copper alloys are strong and hard and do not create sparks when they strike a steel surface. In structural applications, the combination of high flexural rigidity, thermal stability, thermal conductivity and low density (1.85 times that of water) make beryllium metal a desirable aerospace material for aircraft components, missiles, spacecraft, and satellites. [10] Because of its low density and atomic mass, beryllium is relatively transparent to X-rays and other forms of ionizing radiation; therefore, it is the most common window material for X-ray equipment and components of particle physics experiments [10]. The high thermal conductivities of beryllium and beryllium oxide have led to their use in thermal management applications.

\subsection{Lithium}

Lithium (from Greek: l'1q oc, lithos, "stone") is a chemical element with symbol $\mathrm{Li}$ and atomic number 3. It is a soft, silver-white metal belonging to the alkali metal group of chemical elements. Under standard conditions it is the lightest metal and the least dense solid element. Like all alkali metals, lithium is highly reactive and flammable. For this reason, it is typically stored in mineral oil. When cut open, it exhibits a metallic luster, but contact with moist air corrodes the surface quickly to a dull silvery gray, then black tarnish. Because of its high reactivity, lithium never occurs freely in nature, and instead, only appears in compounds, which are usually ionic. Lithium occurs in a number of pegmatitic minerals, but due to its solubility as an ion, is present in ocean water and is commonly obtained from brines and clays. On a commercial scale, lithium is isolated electrolytically from a mixture of lithium chloride and potassium chloride. [2]

Lithium and its compounds have several industrial applications, including heat-resistant glass and ceramics, lithium grease lubricants, flux additives for iron, steel and aluminum production, lithium batteries and lithium-ion batteries. These uses consume more than three quarters of lithium production. [2]

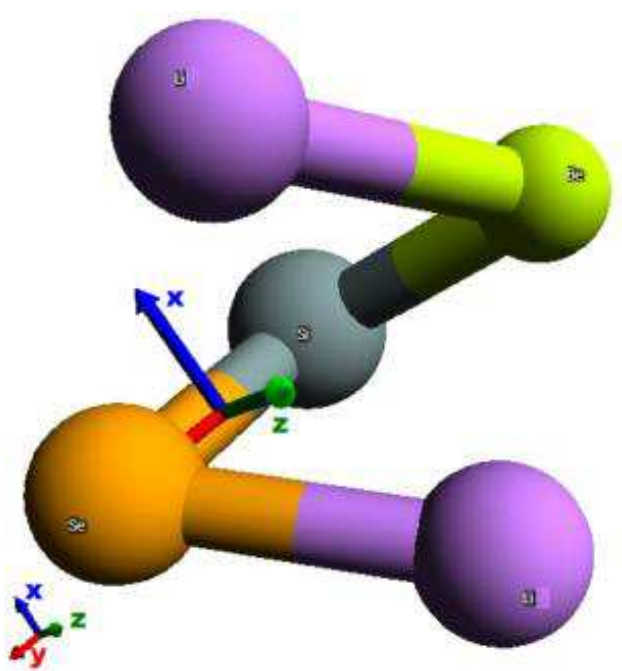

Figure 1. Above and to the left the representation of the molecular structure of BeLi $i_{2} S e S i$ seed [9], obtained through computer via Molecular Mechanics Mm+ calculation, and then its geometry was optimized via PM3 [12, 13, 14, 15, 16] with distance measured in Angstron obtained using computer programs Hyper Chem 7.5 Evaluation [6]. Above and to the right the representation of the molecular structure of BeLi $i_{2} S e S i$, obtained through computer via Ab Initio calculation method DFT, functional B3LYP in base 6-311G**(3df, 3pd), obtained using computer programs GAMESS [7, 17]. Images obtained in the software Mercury 3.8 [18], above and to the left and the right Avogadro [19]. Represented in gray color the atom of Silicon, in the purple color Lithium, in the lemon yellow color Beryllium, and in the pumpkin the Selenium. 


\subsection{Selenium}

Selenium is a chemical element with symbol Se and atomic number 34. It is a nonmetal with properties that are intermediate between those of its periodic table columnadjacent chalcogen elements sulfur and tellurium. It rarely occurs in its elemental state in nature, or as pure ore compounds. Selenium (Greek selene meaning "Moon") was discovered in 1817 by J. Jacob Berzelius. [2]

Selenium is found impurely in metal sulfide ores, copper where it partially replaces the sulfur. The chief commercial uses for selenium today are in glassmaking and in pigments. Selenium is a semiconductor and is used in photocells. Uses in electronics, once important, have been mostly supplanted by silicon semiconductor devices. Selenium continues to be used in a few types of DC power surge protectors and one type of fluorescent quantum dot. [2]

\subsection{Silicon}

Silicon is a chemical element with symbol $\mathrm{Si}$ and atomic number 14. It is a tetravalent metalloid, more reactive than germanium, the metalloid directly below it in the table. [2] Is the eighth most common element in the universe by mass, but very rarely occurs as the pure free element in nature. It is most widely distributed in dusts, sands, planetoids, and planets as various forms of silicon dioxide (silica) or silicates. Over $90 \%$ of the Earth's crust is composed of silicate minerals, making silicon the second most abundant element in the Earth's crust (about $28 \%$ by mass) after oxygen. [11]

Elemental silicon also has a large impact on the modern world economy. Although most free silicon is used in the steel refining, aluminium-casting, and fine chemical industries (often to make fumed silica), the relatively small portion of very highly purified silicon that is used in semiconductor electronics $(<10 \%)$ is perhaps even more critical. Because of wide use of silicon in integrated circuits, the basis of most computers, a great deal of modern technology depends on it. [2]

\section{Methods}

\subsection{Hartree-Fock}

The Hartree-Fock self-consistent method is based on the one-electron approximation in which the motion of each electron in the effective field of all the other electrons is governed by a one-particle Schrodinger" equation. The Hartree-Fock approximation takes into account of the correlation arising due to the electrons of the same spin, however, the motion of the electrons of the opposite spin remains uncorrelated in this approximation. The methods beyond self-consistent field methods, which treat the phenomenon associated with the many-electron system properly, are known as the electron correlation methods. One of the approaches to electron correlation is the Møller-Plesset
(MP) perturbation theory in which the Hartree-Fock energy is improved by obtaining a perturbation expansion for the correlation energy. [20] However, MP calculations are not variational and can produce an energy value below the true energy. [21]

Another first principles approach to calculate the electronic structure for many-electron systems is the Density Functional Theory (DFT). In this theory, the exchangecorrelation energy is expressed, at least formally, as a functional of the resulting electron density distribution, and the electronic states are solved for self-consistently as in the Hartree-Fock approximation. [22, 23, 24, 25] The Density Functional Theory is, in principle, exact but, in practice, both exchange and dynamic correlation effects are treated approximately. [26]

A hybrid exchange-correlation functional is usually constructed as a linear combination of the Hartree-Fock exact exchange functional,

$$
E_{X}^{H F}=-\frac{1}{2} \sum_{i, j} \iint \Psi_{i}^{*}\left(\boldsymbol{r}_{1}\right) \Psi_{j}^{*}\left(\boldsymbol{r}_{1}\right) \frac{1}{r_{12}} \psi_{i}\left(\boldsymbol{r}_{2}\right) \psi_{j}\left(\boldsymbol{r}_{2}\right) d \boldsymbol{r}_{1} d \boldsymbol{r}_{2}
$$

and any number of exchange and correlation explicit density functionals. The parameters determining the weight of each individual functional are typically specified by fitting the functional's predictions to experimental or accurately calculated thermochemical data, although in the case of the "adiabatic connection functionals" the weights can be set a priori. [27]

\subsection{B3LYP}

The B3LYP (Becke, three-parameter, Lee-Yang-Parr) [28, 29] exchange-correlation functional is:

$$
\begin{gathered}
E_{X C}^{B 3 L Y P}=E_{X}^{L D A}+a_{0}\left(E_{X}^{H F}-E_{X}^{L D A}\right)+a_{x}\left(E_{X}^{G G A}-E_{X}^{L D A}\right)+ \\
E_{C}^{L D A}+a_{c}\left(E_{c}^{G G A}-E_{c}^{L D A}\right)
\end{gathered}
$$

Are generalized gradient approximations: the Becke 88 exchange functional [30] and the correlation functional of Lee, Yang and Parr [31] for B3LYP, and $\mathrm{E}_{\mathrm{c}}{ }^{\mathrm{DA}}$ is the VWN local-density approximation to the correlation functional. [32]

The three parameters defining B3LYP have been taken without modification from Becke's original fitting of the analogous B3PW91 functional to a set of atomization energies, ionization potentials, proton affinities, and total atomic energies. [33]

The first principles methods (i.e. HF and DFT) discussed above can be implemented with the aid of the GAMESS set of programs to study the electronic structure and to determine the various physical properties of many-electron systems. [7] A basis set is the mathematical description of the orbitals within a system (which in turn combine to approximate the total electronic wavefunction) used to perform the theoretical calculation. [34] 3-21G, 3-21G*, 6-31G, 6-31G*, 6-31G**, $6-311 \mathrm{G}, 6-311 \mathrm{G}^{*}, 6-311 \mathrm{G}^{* *}$ are the basis sets used in the calculations. The functional Becke-style one parameter 
functional using modified Perdew-Wang exchange and Perdew-Wang 91 correlation is used for DFT Calculations. $[26,35]$

The SCF method and extensions to it are mathematically and physically considerably more complicated than the oneelectron methods already discussed. Thus, one normally does not perform such calculations with pencil and paper, but rather with complicated computer programs. Terms like "Hartree-Fock", or "correlation energy" have specific meanings and are pervasive in the literature. [36]

The vast literature associated with these methods suggests that the following is a plausible hierarchy:

$$
\mathrm{HF}<<\mathrm{MP} 2<\mathrm{CISD}<\mathrm{CCSD}<\mathrm{CCSD}(\mathrm{T})<\mathrm{FCI}
$$

The extremes of 'best', FCI, and 'worst', HF, are irrefutable, but the intermediate methods are less clear and depend on the type of chemical problem being addressed. [37]

For calculations a cluster of 6 computer models was used: Prescott-256 Celeron D processors, [2] featuring double the L1 cache (16 KB) and L2 cache (256 KB), Socket 478 clock speeds of $2.13 \mathrm{GHz}$; Memory DDR2 PC4200 512MB; Hitachi HDS728080PLAT20 80 GB and CD-R.

The dynamic was held in Molecular Mechanics Force Field $(\mathrm{Mm}+)$, Eq. (1), after the quantum computation was optimized via PM3 and then by DFT, $[21,26]$ with functional B3LYP [38] and base 6-311G** [7, 21, 26]. The molecular dynamics at algorithm Polak-Ribiere [39], conjugate gradient, at the termination condition: RMS gradient [40] of $0,1 \mathrm{kcal} / \mathrm{A}$. mol or 405 maximum cycles in vacuum [6].

The first principles calculations have been performed to study the equilibrium configuration of $\mathrm{BeLi}_{2} \mathrm{SeSi}$ molecule using the Hyperchem 7.5 Evaluation [41], Mercury 3.8 a general molecular and electronic structure processing program [18], Avogadro: an advanced semantic chemical editor, visualization, and analysis platform [19] and GAMESS is a computational chemistry software program and stands for General Atomic and Molecular Electronic Structure System [7] set of programs. The first principles approaches can be classified into two main categories: the Hartree-Fock approach and the density functional approach. [22]

\section{Discussions}

The $\mathrm{BeLi}_{2} \mathrm{SeSi}$ molecular has:

Chemical formula: $\mathrm{BeLi}_{2} \mathrm{SeSi}$

Molecular mass: $130.93764 \mathrm{amu}$

Crystal system: triclinic

Density: $215.757 \mathrm{~g} / \mathrm{cm}^{3}$

Wyckoff sequence: a5

A covalent bonding structure between the atoms was initially suggested as shown in Figure (1). Selenium valences +6 , Silicon +4 , Beryllium +2 and Lithium +1 were considered as valences. Selenium binding to the silicon in triple bond, and this to Beryllium in simple bond. Two lithium and beryllium atoms and simple covalent bond to

\section{Selenium.}

The structure of the molecule was initially parameterized and made to the optimization of its geometry using Molecular Mechanics $\mathrm{Mm}^{+}$. [42] After optimization of the geometry via $\mathrm{Mm}+$, it was optimized via PM3 [12, 13, 14, $15,16]$, Figure (1), being the software used was the Hyper Chem 7.5 Evaluation [6]. Above the center and to the right of Figure (1), the molecular structure of the $\mathrm{BeLi}_{2} \mathrm{SeSi}$ molecule was obtained by DFT [21] in the UB3LYP/6-311G**(3df, 3pd) using the GAMESS software. [7]

A detailed analysis of the molecular structure of the $\mathrm{BeLi}_{2} \mathrm{SeSi}$ molecule was obtained using the GAMESS software [7]. We obtained the dipole moment and vibration frequencies of the $\mathrm{BeLi}_{2} \mathrm{SeSi}$ molecule through ab initio calculations by the B3LYP, HF, MP2, CISD, CCSD, $\operatorname{CCSD}(\mathrm{T})$ and FCI methods [21], Tables (3) and (6).

We represent the $\mathrm{BeLi}_{2} \mathrm{SeSi}$ molecule in this sequence of Figure (3) for a better understanding of its three-dimensional structure.

Figure (3) contains 16 images of the molecular structure of $\mathrm{BeLi}_{2} \mathrm{SeSi}$ molecule in different positions under-rotation of the $\mathrm{x}$ and $\mathrm{y}$ axes. From the first image at the top left, a sequence of 16 images in turn sequence is displayed, from left to right and from top to bottom. The molecule is rotated in the $\mathrm{x}$-direction from the first image until it returns to its initial position in the ninth image. From which you rotate $y$ until you return to the starting position, last image in the lower right corner. The atoms are represented in four different colors. Silicon in golden color, Selenium in orange, Beryllium in lime-green and two lithium atoms in purple.

Analyzing Figures (1) and Figure (2) obtained by quantum chemistry by the $a b$ initio method, we can see the structure of the simulated molecule. Making the necessary turns of visualization with the atom of $\mathrm{Be}$ in front of the screen, or the atom of Se, and centralizing these in a segment of line between them, Be and Se, these form a tetrahedron each. Six planes are formed, with six triangles belonging to these planes formed by the atoms, occupying its vertices: Be-Si-Li2, Be-Si-Li1 and Be-Li1-Li2 for the tetrahedron of the atom Beryllium, and Se-Si-L1, Se-Si-Li2 and Se-Li1-Li2 to the tetrahedron of the Selenium atom. Both tetrahedrons connected to the same base, formed by the plane, with the atoms Si-Li1-Li2, as vertices of the base triangle of these.

The covalent bonds between the atoms of the simulated molecule have now changed with the covalent bonding sequence Li2-Be-Si-Se-Li1, i.e. Li-Be-Si-Se-Li, Figure (2), (in the center and the left).

In Tables (1) and (2) the pdb files of the simulated molecule are displayed. The Table (1) the pdb file of the molecular structure of molecule $\mathrm{BeLi}_{2} \mathrm{SeSi}$, obtained through computer via ab initio calculation method HF in base STO$3 \mathrm{G}[43,44]$, obtained using computer programs Hyper Chem 7.5 Evaluation [6]. The Table (2) the pdb file of the molecular structure of molecule $\mathrm{BeLi}_{2} \mathrm{SeSi}$, obtained through computer via ab initio calculation method B3LYP in base 6- 
$311 \mathrm{G}^{* *}(3 \mathrm{df}, 3 \mathrm{pd})$, obtained using computer programs GAMESS [7].

The Table (3) presents some thermochemical properties of the simulated molecule, such as: thermal energy, vaporization heat and molar entropy. All values obtained after a end optimization of the molecular geometry, using the DFT method, with B3LYP functional and base 6$311 \mathrm{G}^{* *}(3 \mathrm{df}, 3 \mathrm{pd})$. The thermochemical values for the HF, MP2, CISD, CCSD(T), CC, CID and CI methods were calculated at the end geometry point B3LYP/6-311G**(3df, $3 \mathrm{pd})$. [21]

The lowest and highest thermal energy obtained were $7,622 \mathrm{kcal} / \mathrm{mol}$ and $8,560 \mathrm{kcal} / \mathrm{mol}$ in the $\mathrm{HF} / 3-21 \mathrm{G}$ and MP2/STO-3G methods, respectively.

The lowest and highest heat of vaporization were 18.592 $\mathrm{kcal} / \mathrm{mol} . \mathrm{K}$ and $20.718 \mathrm{kcal} / \mathrm{mol} . \mathrm{K}$ in the $\mathrm{HF} / 3-21 \mathrm{G}$ and B3LYP/6-311G** (3df, 3pd) methods, respectively.

The lowest and highest molar entropy obtained were $80.298 \mathrm{kcal} / \mathrm{mol} . \mathrm{K}$ and $84.426 \mathrm{kcal} / \mathrm{mol} . \mathrm{K}$, in the $\mathrm{HF} / 6$ $311 \mathrm{G}^{* *}$ (3df, 3pd) and CIS-FC/CC-PVDZ methods, respectively.

The Tables (4) and (5) present the parameters of the molecular geometry of the simulated molecule, such as spatial coordinates, length of the bonds between the atoms, angles and dihedral. The Table (1) shows the values for the dynamics via the HF method in the STO-3G base and Table (2) for the DFT method, functional B3LYP in base 6$311 \mathrm{G} * *(3 \mathrm{df}, 3 \mathrm{pd})$.

The Figures (1) and (2), to the left of these, the representation of the molecular structure of $\mathrm{BeLi}_{2} \mathrm{SeSi}$ seed $[8,9,45,46]$, obtained through computer via Molecular Mechanics $\mathrm{Mm}+$ calculation, and then its geometry was optimized via PM3 $[12,13,14,15,16]$ and the representation of the molecular structure of $\mathrm{BeLi}_{2} \mathrm{SeSi}$ seed, obtained through computer via $a b$ initio calculation method HF in base STO-3G [43, 44], with distance measured in Ångstron and loads obtained using computer programs Hyper Chem 7.5 Evaluation [6], respectively.

There is a reduction of the average distance between atoms of the simulated molecule.

Through the calculations presented in Table (6) show that the lowest and highest electric dipole moment, have the values 0.9869 Debye and 8.9298 Debye, in the B3LYP/STO$3 \mathrm{G}$ and $\mathrm{UHF} / \mathrm{TVZ}$ methods, respectively.

\section{Conclusions}

As a result of the likely molecular structure of the arrangement of a crystal was obtained. The techniques of micro-crushing and conoscopic [47] analysis can lead to evidence and obtaining such crystals.

Calculations using quantum chemistry admit inorganic molecule $\mathrm{BeLi}_{2} \mathrm{SeSi}$.

As a result of calculations $a b$ initio the molecular structure with a feature proposed by the simulated seed molecule, it is likely by quantum chemistry that a crystal structure of the arrangement can be obtained.

The simulated molecule has a cationic molecular structure, in the atoms Selenium and Silicon. As a consequence, it has a strong electric dipole moment, 8.9298 Debye, in UHF/TVZ methods. Due to its geometry, it presents a probable formation structure of a crystal with the tetrahedral and hexahedral crystal structure.

\section{Tables and Figures}

Table 1. Description of the pdb file of the molecular structure of molecule $\mathrm{BeLi}_{2} \mathrm{SeSi}$, obtained through computer via ab initio calculation method $\mathrm{HF}$ in base STO-3G [43, 44], obtained using computer programs Hyper Chem 7.5 Evaluation [6].

\begin{tabular}{lllllll}
\hline HETATM & 1 & $\mathrm{Si}$ & 1 & -0.892 & 1.300 & 0.917 \\
HETATM & 2 & $\mathrm{Se}$ & 2 & -0.010 & -1.387 & -0.224 \\
HETATM & 3 & $\mathrm{Be}$ & 3 & -1.646 & -0.283 & -0.066 \\
HETATM & 4 & $\mathrm{Li}$ & 4 & 0.494 & 0.829 & -0.850 \\
HETATM & 5 & $\mathrm{Li}$ & 5 & -0.199 & -0.606 & 1.992 \\
CONECT & 1 & 2 & 3 & & & \\
CONECT & 2 & 1 & 3 & 4 & 5 & \\
CONECT & 3 & 1 & 2 & & & \\
CONECT & 4 & 2 & & & & \\
CONECT & 5 & 2 & & & & \\
END & & & & & & \\
\hline
\end{tabular}

Table 2. Description of the. pdb file of the molecular structure of molecule $\mathrm{BeLi}_{2} \mathrm{SeSi}$, obtained through computer via ab initio calculation method B3LYP in base 6-311G**(3df, 3pd), obtained using computer programs GAMESS [7].

\begin{tabular}{lllllll}
\hline HETATM & 1 & $\mathrm{Se}$ & 1 & 0.883 & -0.055 & 0.000 \\
HETATM & 2 & $\mathrm{Li}$ & 2 & -0.319 & 1.382 & -1.484 \\
HETATM & 3 & $\mathrm{Si}$ & 3 & -1.359 & -0.805 & 0.000 \\
HETATM & 4 & $\mathrm{Li}$ & 4 & -0.319 & 1.382 & 1.484 \\
HETATM & 5 & $\mathrm{Be}$ & 5 & -2.273 & 1.212 & 0.000 \\
CONECT & 1 & 2 & 3 & & & \\
CONECT & 2 & 1 & & & & \\
CONECT & 3 & 1 & 5 & & & \\
CONECT & 4 & 5 & & & & \\
CONECT & 5 & 3 & 4 & & & \\
END & & & & & & \\
\hline
\end{tabular}

Table 3. Thermochemical parameters of the molecule BeLi $i_{2} S e S i$ obtained by ab initio methods.

\begin{tabular}{llll}
\hline Methods/Base & & & Thermochemistry \\
\hline & ${ }^{\mathbf{E}}$ Thermal & CV & S \\
\hline B3LYP/6-311G**(3df, 3pd) & (Kcal/mol) & (cal/mol.K) & \\
CIS-FC/CC-pVDZ & 8.152 & 20.718 & 83.606 \\
HF/3-21G & 8.523 & 19.714 & 80.298 \\
HF/6-311G**(3df, 3pd) & 7.622 & 18.592 & 82.660 \\
\hline
\end{tabular}




\begin{tabular}{llll}
\hline Methods/Base & & & Thermochemistry \\
\hline & ${ }^{\text {E}}$ Thermal & CV & S \\
\hline & (Kcal/mol) & (cal/mol.K) & (cal/mol.K) \\
\hline MP2/STO-3G & 8.560 & 19.648 & 80.457 \\
CISD/STO-3G & 8.552 & 19.662 & 80.457 \\
CCSD(T)/STO-3G & 8.518 & 19.749 & 80.714 \\
CI/STO-3G & 8.552 & 19.662 & 80.457 \\
B3LYP/STO-3G & 8.438 & 19.944 & 80.979 \\
CC/STO-3G & 8.545 & 19.686 & 80.609 \\
CID/STO-3G & 8.560 & 19.645 & 80.445 \\
\hline
\end{tabular}

Table 4. Molecular parameters of the atoms of the molecule BeLi $i_{2} S e S i$ seed, obtained through computer via ab initio calculation method HF in base STO-3G [43, 44], with distance measured in Angstron obtained using computer programs Hyper Chem 7.5 Evaluation [6].

\begin{tabular}{|c|c|c|c|c|c|c|c|c|c|}
\hline Atom & NA & NB & $\mathrm{NC}$ & Bond & Angle & Dihedral & $\mathbf{X}(\mathbf{A})^{\circ}$ & $Y(A)^{\circ}$ & $\mathbf{Z}(\mathbf{A})^{\circ}$ \\
\hline $\mathrm{Si}$ & & & & & & & $-0,379$ & $-1,080$ & 1,707 \\
\hline $\mathrm{Se}$ & 1 & & & 3,0272777 & & & $-0,671$ & 0,681 & $-0,738$ \\
\hline $\mathrm{Be}$ & 2 & 1 & & 1,8506712 & 40,6512698 & & $-1,661$ & $-0,539$ & 0,240 \\
\hline $\mathrm{Li}$ & 1 & 3 & 2 & 2,4351417 & 69,0527075 & 53,4172985 & $-0,624$ & 1,341 & 1,614 \\
\hline $\mathrm{Li}$ & 3 & 2 & 1 & 2,4492640 & 68,7308859 & $-58,1835086$ & 0,412 & $-1,542$ & $-0,594$ \\
\hline
\end{tabular}

Table 5. Molecular parameters of the atoms of the molecule BeLi2SeSi seed, obtained through computer via ab initio calculation method DFT, functional B3LYP in base 6-311G**(3df, 3pd), with distance measured in Angstron obtained using computer programs GAMESS. [7].

\begin{tabular}{lllllllll}
\hline Atom & NA & NB & NC & Bond & Angle & Dihedral & X(A) & Y(A) \\
\hline $\mathrm{Se}$ & & & & & & & 0.883497 & -0.054990 \\
$\mathrm{Li}$ & 1 & & & 2.390969 & & -0.319339 & 1.382430 \\
$\mathrm{Si}$ & 1 & 2 & & 2.364837 & 73.358 & & -1.359205 & -0.805152 \\
$\mathrm{Li}$ & 1 & 3 & 4 & 2.390972 & 73.357 & 40.393 & -0.319392 & 1.382375 \\
$\mathrm{Be}$ & 3 & 3 & 2 & 2.214531 & 95.889 & -40.393 & -2.273460 & 1.484518 \\
\hline
\end{tabular}

Table 6. Table containing the dipole moments of the BeLi $i_{2} S e S i$ molecule via ab initio methods.

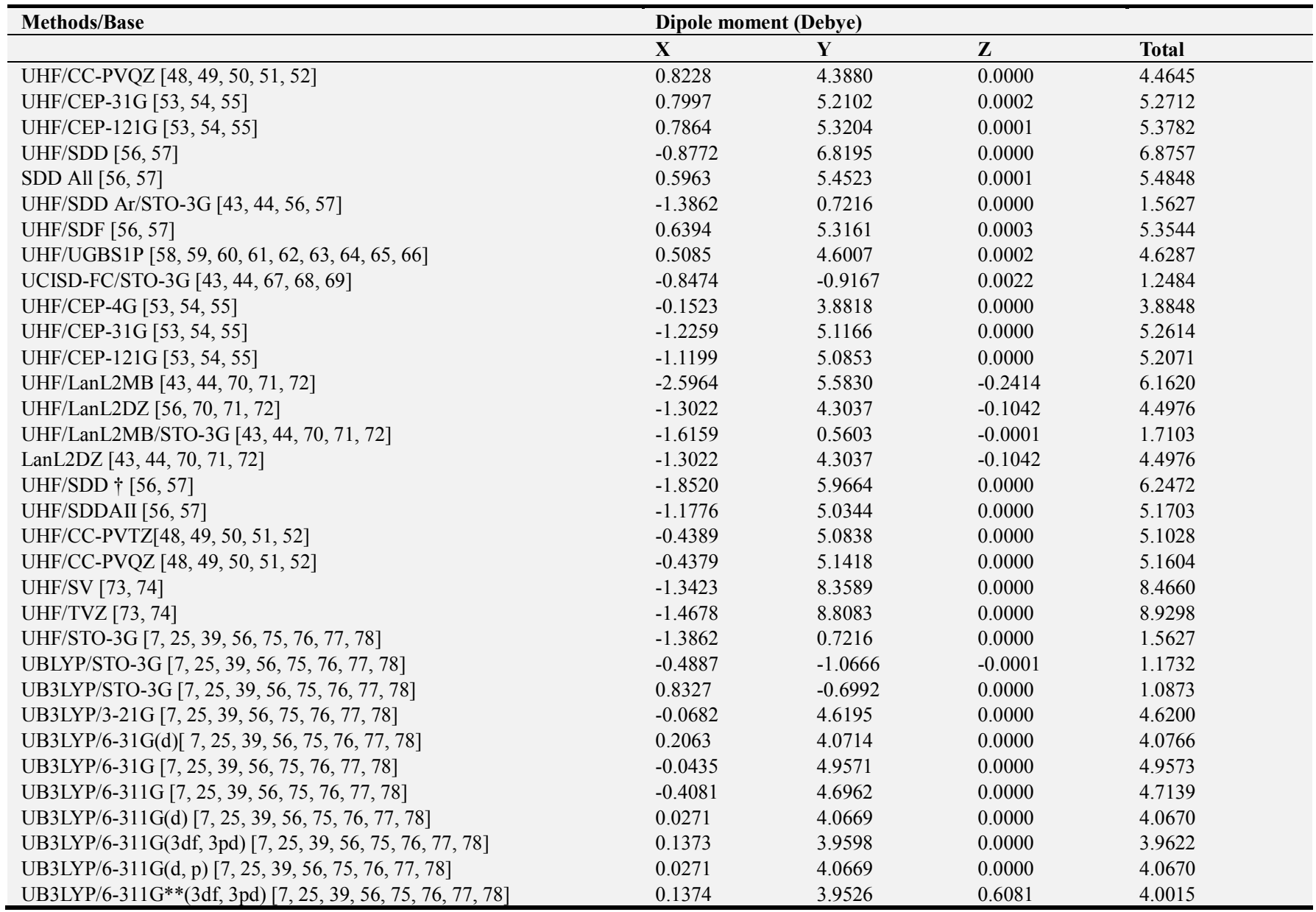




\begin{tabular}{lllll}
\hline Methods/Base & \multicolumn{2}{l}{ Dipole moment (Debye) } & & Total \\
\hline & X & Y & Z & 5.0724 \\
UCIS-FC/CC-PVDZ [48, 49, 50, 51, 52, 79] & -0.5403 & 5.0436 & 0.0000 & 5.0232 \\
UHF/3-21G [7, 25, 39, 56, 75, 76, 77, 78] & -0.8811 & 4.9453 & 0.0000 & 5.1519 \\
UHF/6-311G**(3df, 3pd) [7, 25, 39, 56, 75, 76, 77, 78] & -0.4440 & 5.1327 & 0.0000 & 1.7103 \\
UMP2-FC/STO-3G [43, 44, 80, 81, 82, 83, 84, 85] & -1.6159 & 0.5603 & -0.0001 & 1.7185 \\
UCCSD(T)-FC/STO-3G [43, 44, 86, 87, 88, 89, 90, 91] & -1.6152 & 0.5864 & 0.0181 & 1.2484 \\
UCISD-FC/STO-3G [43, 44, 92, 93, 94] & -0.8474 & -0.9167 & 0.0022 & 0.9869 \\
B3LYP/STO-3G [7, 25, 39, 43, 44, 56, 75, 76, 77, 78] & -0.7344 & -0.6592 & -0.0001 & 1.3670 \\
UCCD-FC/STO-3G [43, 44, 86, 87] & -0.7643 & -1.1334 & 0.0022 & 1.2483 \\
UCID-FC/STO-3G [43, 44, 92, 93, 94] & -0.8846 & -0.8808 & 0.0022 & \\
\hline
\end{tabular}

${ }^{\dagger}$ D95 up to Ar [56] and Stuttgart/Dresden ECPs on the remainder of the periodic table. [57] Selects Stuttgart potentials for Z $>2$.
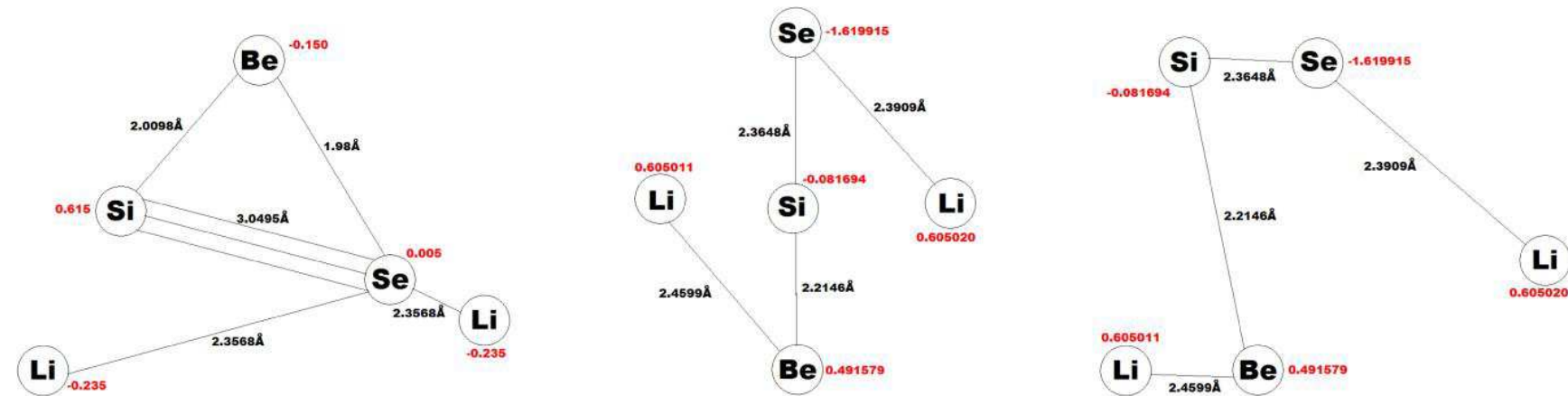

Figure 2. Above and to the left the representation of the molecular structure of BeLi $i_{2} S e S i$ seed, obtained through computer via ab initio calculation method HF in base STO-3G [43, 44], with distance measured in Angstron and loads obtained using computer programs Hyper Chem 7.5 Evaluation [6], after the structure of the molecule was initially parameterized and made to the optimization of its geometry using Molecular Mechanics Mm+. [42] After optimization of the geometry via Mm+, it was optimized via PM3 [12, 13, 14, 15, 16], Figure (1). Above, in the center and to the right the representation of the molecular structure of BeLi $i_{2} \mathrm{SeSi}$, [18] obtained through computer via ab initio calculation method DFT [21], functional B3LYP in base 6-311G**(3df, 3pd), with distance measured in Angstron and loads in units of atomic charge obtained using computer programs GAMESS [7].
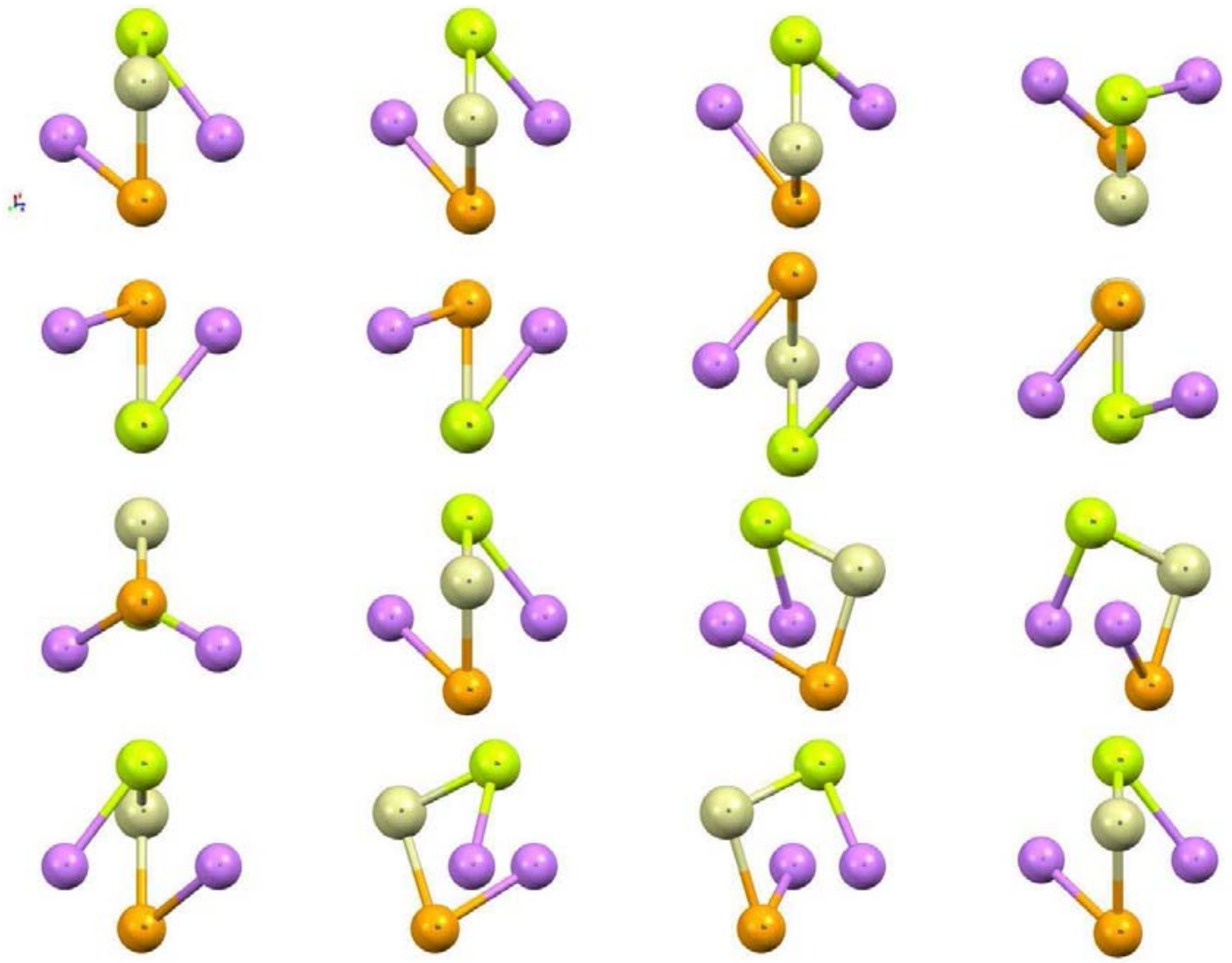

Figure 3. Representation of the molecular structure of BeLi 2 SeSi, [18] obtained through computer via ab initio calculation method DFT, functional B3LYP in base 6-311 G** (3df, 3pd), obtained using computer software GAMESS [7]. Images obtained in the software Mercury 3.8 [18]. Represented in cornsilk color the atom of Silicon, in the purple color Lithium, in the lemon yellow color Beryllium, and in the pumpkin the Selenium. 


\section{References}

[1] R. E. Newnham. Properties of materials. Anisotropy, Simmetry, Structure. New York, 2005.

[2] CC BY-NC-SA 3.0. Creative commons. Wikipedia, The Free Encyclopedia, May 2016. CC BY-NC-SA 3.0.

[3] R. J. Matthys. Crystal Oscillator Circuits. Malabar, Florida $32950,1992$.

[4] C. D. Gribble and A. J. Hall. A Practical Introduction to Optical Mineralogy. 1985.

[5] N. C. Braga. Como funciona o cristal na eletronica^ (art 423). Instituto Newton C. Braga, July 2015.

[6] Computational chemistry software. hyperchem 7.5 evaluation. Hypercube, Inc., 2003.

[7] M. S. Gordon et al. General atomic and molecular electronic structure system (gamess). J. Comput. Chem., 14:1347-1363, 1993.

[8] R. Gobato; A. Gobato and D. F. G. Fedrigo. Inorganic arrangement crystal beryllium, lithium, selenium and silicon. In XIX Semana da F'isica. Simposio' Comemorativo dos 40 anos do Curso de F'isica da Universidade Estadual de Londrina, Rodovia Celso Garcia Cid, Pr 445 Km 380, Campus

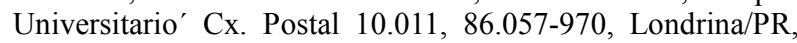
2014. Universidade Estadual de Londrina (UEL).

[9] R. Gobato; A. Gobato and D. F. G. Fedrigo. Inorganic arrangement crystal beryllium, lithium, selenium and silicon. Cornell University Library. arXiv.org, Aug 01 2015. Atomic and Molecular Clusters (physics.atm-clus); Materials Science (cond-mat.mtrl-sci).

[10] Concise encyclopedia chemistry. De Gruyter; Rev Sub edition (February 1994), 1994.

[11] R. Nave. Abundances of the elements in the earth's crust. Georgia State University.

[12] M. J. Frisch; G. Scalmani; T. Vreven and G. Zheng. Analytic second derivatives for semiempirical models based on MNDO. Mol. Phys, 2009.

[13] W. Thiel and A. A. Voityuk. Extension of MNDO to d orbitals: Parameters and results for the second-row elements and for the zinc group. J. Phys. Chem., (100):616-26, 1996.

[14] W. Thiel and A. A. Voityuk. Extension of the MNDO formalism to $\mathrm{d}$ orbitals: Integral approximations and preliminary numerical results. Theor. Chem. Acc., (81):391404, 1992.

[15] J. J. P. Stewart. Optimization of parameters for semiempirical methods. I. Methods. J. Comp. Chem., (10):209-20, 1989.

[16] J. J. P. Stewart. Optimization of parameters for semiempirical methods. II. Applications. J. Comp. Chem., (10):221-64, 1989.

[17] R. Gobato. Study of the molecular geometry of Caramboxin toxin found in star flower (Averrhoa carambola L.). PJSE, 3(1):1-9, January 2017.

[18] The Cambridge Crystallographic Data Centre (CCDC).
Mercury - crystal structure visualisation, exploration and analysis made easy, May 2012. Mercury 3.1 Development (Build RC5).

[19] M. D. Hanwell; D. E. Curtis; D. C. Lonie; T. Vandermeersch; E. Zurek and G. R. Hutchison. Avogadro: An advanced semantic chemical editor, visualization, and analysis platform. Journal of Cheminformatics, 4(16), 2012.

[20] A. Szabo and N. S. Ostlund. Modern Quantum Chemistry. Dover Publications, New York, 1989.

[21] I. N. Levine. Quantum Chemistry. Pearson Education (Singapore) Pte. Ltd., Indian Branch, 482 F. I. E. Patparganj, Delhi 110 092, India, 5th ed edition, 2003.

[22] K. Ohno; K. Esfarjani and Y. Kawazoe. Computational Material Science. Springer-Verlag, Berlin, 1999.

[23] K. Wolfram and M. C. Hothausen. Introduction to DFT for Chemists. John Wiley \& Sons, Inc. New York, 2nd ed edition, 2001.

[24] P. Hohenberg and W. Kohn. Inhomogeneous electron gas. Phys. Rev., (136): B864-B871, 1964.

[25] W. Kohn and L. J. Sham. Self-consistent equations including exchange and correlation effects. Phys. Rev., (140): A1133, 1965.

[26] J. M. Thijssen. Computational Physics. Cambridge University Press, Cambridge, 2001.

[27] J. P. Perdew; M. Ernzerhof and K. Burke. Rationale for mixing exact exchange with density functional approximations. J. Chem. Phys, 105(22):9982-9985, 1996.

[28] K. Kim and K. D. Jordan. Comparison of Density Functional and MP2 Calculations on the Water Monomer and Dimer. J. Phys. Chem., 40(98):10089-10094, 1994.

[29] P. J. Stephens; F. J. Devlin; C. F. Chabalowski and M. J. Frisch. Ab Initio Calculation of Vibrational Absorption and Circular Dichroism Spectra Using Density Functional Force Fields. J. Phys. Chem., 45(98):11623-11627, 1994.

[30] A. D. Becke. Density-functional exchange-energy approximation with correct asymptotic behavior. Phys. Rev. A., 38(6):3098-3100, 1988.

[31] C. Lee; W. Yang and R. G. Parr. Development of the ColleSalvetti correlation-energy formula into a functional of the electron density. Phys. Rev. B, 37(2):785-789, 1988.

[32] S. H. Vosko; L. Wilk and M. Nusair. Accurate spin-dependent electron liquid correlation energies for local spin density calculations: a critical analysis. Can. J. Phys, 58(8):12001211, 1980.

[33] A. D. Becke. Density-functional thermochemistry. The role of exact exchang. J. Chem. Phys., 98(7):5648-5652, 1993.

[34] J. B. Foresman and Æleen Frisch. Exploring Chemistry with Electronic Structure Methods. Gaussian, Inc. Pittsburgh, PA, 2nd ed edition, 1996.

[35] L. Mainali; D. R. Mishra and M. M. Aryal. First principles calculations to study the equilibrium configuration of ozone molecule. Department of Biophysics. Medical College of Wisconsin. 8701 Watertown Plank Road. Milwaukee, WI 53226. 
[36] J. P. Lowe and K. A. Peterson. Quantum Chemistry. Elsevier Inc., third edition edition, 30 Corporate Drive, Suite 400, Burlington, MA 01803, USA; 525 B Street, Suite 1900, San Diego, CA 92101-4495, USA; 84 Theobalds Road, London WC1X 8RR, UK. 2006.

[37] J. J. W. Mc Douall. Computational Quantum Chemistry. Molecular Structure and Properties in Silico. The Royal Society of Chemistry, Thomas Graham House, Science Park, Milton Road, Cambridge CB4 0WF, UK, 2013.

[38] W. Yang C. Lee and R. G. Parr. Phys. Rev. B, 37:785-789, 1988.

[39] E. Polak. Computational Methods in Optimization, volume 77. Elsevier, 111 Fifth Avenue, New York, New York 10003, 1971.

[40] Anthony K. Rappe' and Carla J. Casewit. Molecular Mechanics Across Chemistry. University Science Books, 55D Gate Five Road, Sausalito, CA 94965, 1952(1997).

[41] Hypercube. Hyperchem.7.5 evaluation, 2003. http://www.hyper.com/.

[42] R. Gobato. Benzoca'ina, um estudo computacional. Master's thesis, Universidade Estadual de Londrina (UEL), 2008.

[43] W. J. Hehre; R. F. Stewart and J. A. Pople. Self-Consistent Molecular Orbital Methods. Use of Gaussian expansions of Slater-type atomic orbitals. J. Chem. Phys., (51):2657-64, 1969.

[44] J. B. Collins; P. v. R. Schleyer; J. S. Binkley; and J. A. Pople. Self-Consistent Molecular Orbital Methods. Geometries and binding energies of second-row molecules. A comparison of three basis sets. J. Chem. Phys., (64):5142-51, 1976.

[45] R. Gobato; A. Gobato and D. F. G. Fedrigo. Molecular electrostatic potential of the main monoterpenoids compounds found in oil Lemon Tahiti - (Citrus Latifolia Var Tahiti). Parana Journal of Science and Education, 1(1):1-10, November 2015.

[46] R. Gobato; D. F. G. Fedrigo and A. Gobato. Allocryptopine, Berberine, Chelerythrine, Copsitine, Dihydrosanguinarine, Protopine and Sanguinarine. Molecular geometry of the main alkaloids found in the seeds of Argemone Mexicana Linn. PJSE, 1(2):7-16, December 2015.

[47] A. J. R. Nardy; F. B. Machado; A. Zanardo and T. M. B. Galembeck. Mineralogia Optica de cristais transparentes. Parte pratica. Unesp: Cultura Academica, 2010.

[48] T. H. Dunning Jr. Gaussian basis sets for use in correlated molecular calculations. The atoms boron through neon and hydrogen. J. Chem. Phys., (90):1007-23, 1989.

[49] R. A. Kendall; T. H. Dunning Jr. and R. J. Harrison. Electron affinities of the first-row atoms revisited. Systematic basis sets and wave functions. J. Chem. Phys., (96):6796-806, 1992.

[50] D. E. Woon and T. H. Dunning Jr. Gaussian-basis sets for use in correlated molecular calculations. The atoms aluminum through argon. J. Chem. Phys., (98):1358-71, 1993.

[51] K. A. Peterson; D. E. Woon and T. H. Dunning Jr. Benchmark calculations with correlated molecular wave functions. The classical barrier height of the $\mathrm{H}+\mathrm{H} 2-i \mathrm{H} 2+\mathrm{H}$ reaction. J. Chem. Phys., (100):7410-15, 1994.

[52] A. K. Wilson; T. van Mourik and T. H. Dunning Jr. Gaussian basis sets for use in Correlated Molecular Calculations. Sextuple zeta correlation consistent basis sets for boron through neon. J. Mol. Struct. (Theochem), (388):339-49, 1996.

[53] W. J. Stevens; H. Basch and M. Krauss. Compact effective potentials and efficient shared-exponent basis-sets for the 1st-row and 2nd-row atoms. J. Chem. Phys., (81):6026-33, 1984.

[54] W. J. Stevens; M. Krauss; H. Basch and P. G. Jasien. Relativistic compact effective potentials and efficient, sharedexponent basis-sets for the 3rd-row, 4th-row, and 5th-row atoms. Can. J. Chem., (70):612-30, 1992.

[55] T. R. Cundari and W. J. Stevens. Effective core potential methods for the lanthanides. J. Chem. Phys., (98):5555-65, 1993.

[56] T. H. Dunning Jr. and P. J. Hay. in Modern Theoretical Chemistry, volume 3. Plenum, New York, 1977.

[57] P. Fuentealba; H. Preuss; H. Stoll and L. v. Szentpaly'. A Proper Account of Core-polarization with Pseudopotentials Single Valence-Electron Alkali Compounds. Chem. Phys. Lett., pages 418-22, 1982.

[58] D. M. Silver; S. Wilson and W. C. Nieuwpoort. Universal basis sets and transferability of integrals. Int. J. Quantum Chem., (14):635-39, 1978.

[59] D. M. Silver and W. C. Nieuwpoort. Universal atomic basis sets. Chem. Phys. Lett., (15):421-22, 1978.

[60] J. R. Mohallem; R. M. Dreizler and M. Trsic. A griffin-HillWheeler version of the Hartree-Fock equations. Int. J. Quantum Chem., 30(S20):45-55, 1986. Quant. Chem. Symp.

[61] J. R. Mohallem and M. Trsic. A universal Gaussian basis set for atoms $\mathrm{Li}$ through $\mathrm{Ne}$ based on a generator coordinate version of the Hartree-Fock equations. J. Chem. Phys., (86):5043-44, 1987.

[62] H. F. M. da Costa; M. Trsic and J. R. Mohallem. Universal Gaussian and Slater-type basis-sets for atoms He to Ar based on an integral version of the Hartree-Fock equations. Mol. Phys., (62):91-95, 1987.

[63] A. B. F. da Silva; H. F. M. da Costa and M. Trsic. Universal Gaussian and Slater-type bases for atoms $\mathrm{H}$ to Xe based on the generator-coordinate Hartree-Fock method. Ground and certain low-lying excited-states of the neutral atoms. Mol. Phys, (68):433-45, 1989.

[64] F. E. Jorge; E. V. R. de Castro and A. B. F. da Silva. A universal Gaussian basis set for atoms Cerium through Lawrencium generated with the generator coordinate HartreeFock method. J. Comp. Chem., (18):1565-69, 1997.

[65] F. E. Jorge; E. V. R. de Castro and A. B. F. da Silva. Accurate universal Gaussian basis set for hydrogen through lanthanum generated with the generator coordinate Hartree-Fock method. Chem. Phys, (216):317-21, 1997.

[66] E. V. R. de Castro and F. E. Jorge. Accurate universal gaussian basis set for all atoms of the periodic table. J. Chem. Phys., (108):5225-29, 1998.

[67] J. A. Pople; M. Head-Gordon and K. Raghavachari. Quadratic configuration interaction - a general technique for determining electron correlation energies. J. Chem. Phys., (87):5968-75, 1987. 
[68] J. Gauss and D. Cremer. Analytical evaluation of energy gradients in quadratic configuration-interaction theory. Chem. Phys. Lett., (150):280-86, 1988.

[69] E. A. Salter; G. W. Trucks and R. J. Bartlett. Analytic energy derivatives in many-body methods. i. first derivatives. J. Chem. Phys., (90):1752-66, 1989.

[70] P. J. Hay and W. R. Wadt. Ab initio effective core potentials for molecular calculations - potentials for the transition-metal atoms Sc to Hg. J. Chem. Phys., (82):270-83, 1985.

[71] W. R. Wadt and P. J. Hay. Ab initio effective core potentials for molecular calculations - potentials for main group elements Na to Bi. J. Chem. Phys., (82):284-98, 1985.

[72] P. J. Hay and W. R. Wadt. Ab initio effective core potentials for molecular calculations - potentials for $\mathrm{K}$ to $\mathrm{Au}$ including the outermost core orbitals. J. Chem. Phys., (82):299-310, 1985 .

[73] F. Weigend and R. Ahlrichs. Balanced basis sets of split valence, triple zeta valence and quadruple zeta valence quality for $\mathrm{H}$ to Rn: Design and assessment of accuracy. Phys. Chem. Chem. Phys., (7):3297-305, 2005.

[74] F. Weigend. Accurate Coulomb-fitting basis sets for $\mathrm{H}$ to $\mathrm{Rn}$. Phys. Chem. Chem. Phys., (8):1057-65, 2006.

[75] E. Eliav. Elementary introduction to Molecular Mechanics and Dynamics, Jun 2013.

[76] W. J. Hehre. A Guide to Molecular Mechanics and Quantum Chemical Calculations, Wavefunction. Inc., Irvine, CA, 2003.

[77] M. S. Gordon and M. W. Schmidt. Advances in electronic structure theory: GAMESS a decade later. Theory and Applications of Computational Chemistry: the first forty years. Elsevier. C. E. Dykstra, G. Frenking, K. S. Kim and G. E. Scuseria (editors), pages 1167-1189, 2005. Amsterdam.

[78] R. G. Parr and W. Yang. Density Functional Theory. 1989.

[79] J. B. Foresman; M. Head-Gordon; J. A. Pople and M. J. Frisch. Toward a Systematic Molecular Orbital Theory for Excited States. J. Phys. Chem., (96):135-49, 1992.

[80] C. Møller and M. S. Plesset. Note on an approximation treatment for many-electron systems. Phys. Rev., (46):061822,1934

[81] M. J. Frisch; M. Head-Gordon and J. A. Pople. Direct MP2 gradient method. Chem. Phys. Lett., (166):275-80, 1990.

[82] M. J. Frisch; M. Head-Gordon and J. A. Pople. Semi-direct algorithms for the MP2 energy and gradient. Chem. Phys. Lett., (166):281-89, 1990.

[83] M. Head-Gordon; J. A. Pople and M. J. Frisch. MP2 energy evaluation by direct methods. Chem. Phys. Lett., (153):50306, 1988 .

[84] S. Saebø and J. Almløf. Avoiding the integral storage bottleneck in LCAO calculations of electron correlation. Chem. Phys. Lett., (154):83-89, 1989.

[85] M. Head-Gordon and T. Head-Gordon. Analytic MP2 Frequencies Without Fifth Order Storage: Theory and Application to Bifurcated Hydrogen Bonds in the Water Hexamer; Chem. Phys. Lett; (220):122-28, 1994.

[86] R. J. Bartlett and G. D. Purvis III. Many-body perturbationtheory, coupled-pair many-electron theory, and importance of quadruple excitations for correlation problem. Int. J. Quantum Chem., (14):561-81, 1978.

[87] J. A. Pople; R. Krishnan; H. B. Schlegel and J. S. Binkley. Electron correlation theories and their application to the study of simple reaction potential surfaces. Int. J. Quantum Chem; (14):545-60, 1978.

[88] J. C'1vzek. in Advances in Chemical Physics, volume 14. Wiley Interscience, New York, 35, 1969.

[89] G. D. Purvis III and R. J. Bartlett. A full coupled-cluster singles and doubles model - the inclusion of disconnected triples. J. Chem. Phys; (76):1910-18, 1982.

[90] G. E. Scuseria; C. L. Janssen and H. F. Schaefer III. An efficient reformulation of the closed-shell coupled cluster single and double excitation (CCSD) equations. J. Chem. Phys, (89):7382-87, 1988.

[91] G. E. Scuseria and H. F. Schaefer III. Is coupled cluster singles and doubles (CCSD) more computationally intensive than quadratic configuration-interaction (QCISD)? J. Chem. Phys; (90):3700-03, 1989.

[92] J. A. Pople; R. Seeger and R. Krishnan. Variational Configuration Interaction Methods and Comparison with Perturbation Theory. Int. J. Quantum Chem; Suppl. (Y11):149-63, 1977.

[93] K. Raghavachari; H. B. Schlegel and J. A. Pople. Derivative studies in configuration-interaction theory. J. Chem. Phys; (72):4654-55, 1980.

[94] K. Raghavachari and J. A. Pople. Calculation of one-electron properties using limited configuration-interaction techniques. Int. J. Quantum Chem; (20):1067-71, 1981. 